\title{
Une démonstration élémentaire du théorème central limite
}

\author{
Robert C. Dalang ${ }^{1}$
}

Robert Dalang promovierte an der Ecole Polytechnique Fédérale de Lausanne (EPFL) im Jahr 1987. Danach wirkte er für drei Jahre als Assistenzprofessor an der University of California at Berkeley und anschliessend an der Tufts University. Im Jahr 1995 wurde er auf eine Professur in Wahrscheinlichkeitstheorie an der EPFL berufen. Seine Forschungsinteressen liegen im Bereich der stochastischen Prozesse, insbesondere bei stochastischen partiellen Differentialgleichungen sowie der stochastischen Optimierung und Kontrolle.

\section{Introduction}

Dans tout premier cours de Probabilités du cycle universitaire, on énonce le théorème central limite sous la forme suivante.

Théorème 1. Soit $\left(X_{n}, n \in \mathbb{N}\right)$ une suite de variables aléatoires indépendantes et identiquement distribuées telles que $0<\operatorname{Var}\left(X_{1}\right)<+\infty$. Posons $\mu=E\left(X_{1}\right), \sigma^{2}=\operatorname{Var}\left(X_{1}\right)$

\footnotetext{
${ }^{1}$ La recherche de l'auteur est partiellement financée par le Fonds National Suisse pour la Recherche Scientifique.
}

In jedem universitären Einführungskurs zur Wahrscheinlichkeitsrechnung findet der klassische Zentrale Grenzwertsatz zumindest seine Formulierung, sein gängiger Beweis (mit Hilfe charakteristischer Funktionen) erfolgt aber in der Regel erst in einem fortgeschrittenen Kursabschnitt. Im vorliegenden Beitrag wird ein elementarer Beweis dieses grundlegenden Satzes der Wahrscheinlichkeitstheorie für unabhängige, identisch verteilte Zufallsgrössen unter der Annahme der Existenz der dritten Momente vorgestellt. Dieser von Lindeberg aus dem Jahr 1922 stammende Beweis kann in einem Einführungskurs geführt werden. Er kommt gänzlich ohne Masstheorie aus und benutzt nur elementare Fakten aus einem Einführungskurs in die Analysis. Mit einer etwas subtileren Argumentation führt die verwendete Methode zu einem vollständigen Beweis des Zentralen Grenzwertsatzes unter der gängigen Annahme der Existenz der zweiten Momente. 
et $S_{n}=X_{1}+\cdots+X_{n}$. Alors pour tout $x \in \mathbb{R}$,

$$
\lim _{n \rightarrow \infty} P\left\{\frac{S_{n}-n \mu}{\sigma \sqrt{n}} \leq x\right\}=\frac{1}{\sqrt{2 \pi}} \int_{-\infty}^{x} e^{-u^{2} / 2} d u .
$$

Généralement, la démonstration de ce théorème est repoussée aux cours de probabilités plus avancés [13], ou alors une démonstration incomplète est proposée: on montre la convergence de la fonction génératrice des moments (ce qui requiert une hypothèse sur l'existence des moments exponentiels de $X_{1}$ ), puis on cite, sans démonstration, le fait que cette convergence implique la convergence des fonctions de répartition $[4,8,11]$; deux exceptions sont Sanz-Solé [12] et Krengel [7]. Cette manière de faire convient bien aux étudiants principalement concernés par les applications des probabilités, mais se révèle peu satisfaisante pour des étudiants intéressés par ses aspects mathématiques.

Dans cette note, nous présentons d'abord une démonstration élémentaire et complète du théorème central limite, sous la seule hypothèse supplémentaire suivante.

Hypothèse A. $E\left(\left|X_{1}\right|^{3}\right)<\infty$.

Cette démonstration, due à Lindeberg [9], ne requiert aucune connaissance de la théorie de la mesure, mais uniquement les concepts présentés traditionnellement dans un premier cours de probabilités: premières propriétés de l'espérance, indépendance, loi gaussienne, (plus précisément, linéarité et monotonie de l'espérance, l'espérance du produit de deux variables aléatoires (v.a.) indépendantes est le produit de leur espérances et la propriété d'additivité des v.a. gaussiennes indépendantes). Elle requiert aussi des connaissances de base en analyse, qui sont généralement enseignées avant les probabilités, en particulier la formule de Taylor et les notions de lim sup et lim inf. Notre rédaction est conçue pour être accessible aux étudiants.

Dans une deuxième partie, nous présentons une estimation de la différence, pour $n$ fixé, entre la fonction de répartition de $S_{n}$ et celle de la loi normale standard, ce qui donne en plus une estimation de la vitesse de convergence de la probabilité dans le membre de gauche de (1) vers le membre de droite. Cette estimation ne demande pas de connaissances mathématiques supplémentaires, mais présente plusieurs subtilités qui ne seront sans doute qu'à la portée des étudiants les plus motivés.

Dans une troisième partie, nous présentons la démonstration du théorème 1 sans hypothèse supplémentaire. Cette démonstration, toujours due à Lindeberg [9], exige un peu plus de maturité mathématique que la précédente. Au niveau formel, elle utilise le théorème de la convergence monotone, dont on peut se passer par une vérification directe si on se limite aux v.a. discrètes et absolument continues.

Il me semble que ces démonstrations méritent d'être plus largement connues et j'ai été surpris par le fait que peu de livres en parlent. Elles sont bien connues des spécialistes du théorème central limite pour les v.a. à valeurs dans les espaces de Banach ([1, Chap. 1] et [3]), car la méthode de Lindeberg s'étend à ce niveau de généralité. Divers raffinements se trouvent par exemple dans $[10, \S 1.3]$.

Remerciements. L'auteur remercie Marta Sanz-Solé de lui avoir montré son livre [12], Ch.E. Pfister de lui avoir communiqué ses notes de cours sur la démonstration de Lindeberg, et S.D. Chatterji pour les discussions intéressantes et les références qu'il m'a signalées. 


\section{Démonstration du théorème 1 sous l'hypothèse $\mathrm{A}$}

La démonstration est découpée en quatre étapes, dont la première est une utilisation astucieuse de la formule de Taylor. On notera $C_{\mathrm{b}}^{3}(\mathbb{R})$ l'ensemble des fonctions de $\mathbb{R}$ dans $\mathbb{R}$ bornées de classe $C^{3}$ dont les trois premières dérivées sont bornées.

Lemme 2. Soit $g \in C_{\mathrm{b}}^{3}(\mathbb{R}), V, Y$ et $Z$ trois v.a. indépendantes telles que $E(Y)=E(Z)$ et $E\left(Y^{2}\right)=E\left(Z^{2}\right)$. Posons $C=\sup _{x \in \mathbb{R}}\left|g^{(3)}(x)\right|$. Alors

$$
|E(g(V+Y))-E(g(V+Z))| \leq \frac{C}{3 !}\left(E\left(|Y|^{3}\right)+E\left(|Z|^{3}\right)\right) .
$$

Démonstration. Rappelons la formule de Taylor d'ordre 2: pour tous $x, h \in \mathbb{R}$,

où

$$
g(x+h)=g(x)+g^{\prime}(x) h+\frac{1}{2} g^{\prime \prime}(x) h^{2}+R_{2}(x, h),
$$

$$
R_{2}(x, h)=\frac{1}{2 !} \int_{x}^{x+h}(x+h-t)^{2} g^{(3)}(t) d t .
$$

En particulier,

$$
\left|R_{2}(x, h)\right| \leq \frac{1}{3 !} C|h|^{3} .
$$

D'après (2),

$$
E(g(V+Y))=E(g(V))+E\left(g^{\prime}(V) Y\right)+\frac{1}{2} E\left(g^{\prime \prime}(V) Y^{2}\right)+E\left(R_{2}(V, Y)\right) .
$$

Vu que $V$ et $Y$ sont indépendantes, ceci se réécrit

$$
E(g(V+Y))=E(g(V))+E\left(g^{\prime}(V)\right) E(Y)+\frac{1}{2} E\left(g^{\prime \prime}(V)\right) E\left(Y^{2}\right)+E\left(R_{2}(V, Y)\right) .
$$

De même,

$$
E(g(V+Z))=E(g(V))+E\left(g^{\prime}(V)\right) E(Z)+\frac{1}{2} E\left(g^{\prime \prime}(V)\right) E\left(Z^{2}\right)+E\left(R_{2}(V, Z)\right)
$$

En faisant la différence de (4) et (5) et en utilisant l'égalité des deux premiers moments de $Y$ et $Z$, on obtient

$$
\begin{aligned}
|E(g(V+Y))-E(g(V+Z))| & =\left|E\left(R_{2}(V, Y)\right)-E\left(R_{2}(V, Z)\right)\right| \\
& \leq E\left(\left|R_{2}(V, Y)\right|\right)+E\left(\left|R_{2}(V, Z)\right|\right) .
\end{aligned}
$$

D'après (3), le membre de droite est majoré par $\frac{C}{3 !}\left(E\left(|Y|^{3}\right)+E\left(|Z|^{3}\right)\right)$, ce qui démontre le lemme 2.

La deuxième étape contient l'idée principale de la démonstration et nous rapproche de la conclusion du théorème 1 . Elle traite de la convergence de l'espérance d'une fonction lisse d'une somme normalisée de v.a.

Lemme 3. Soit $\left(\tilde{Y}_{n}, n \in \mathbb{N}\right)$ une suite de v.a. i.i.d. telles que $E\left(\left|\tilde{Y}_{1}\right|^{3}\right)<\infty, E\left(\tilde{Y}_{1}^{2}\right)=1$ et $E\left(\tilde{Y}_{1}\right)=0$. Soit $\tilde{Z}$ une v.a. de loi $N(0,1)$. Alors pour toute fonction $g \in C_{\mathrm{b}}^{3}(\mathbb{R})$,

$$
\lim _{n \rightarrow \infty} E\left(g\left(\frac{\tilde{Y}_{1}+\cdots+\tilde{Y}_{n}}{\sqrt{n}}\right)\right)=E(g(\tilde{Z}))
$$


Démonstration. Fixons $g \in C_{\mathrm{b}}^{3}(\mathbb{R})$ et $n \in \mathbb{N}$. Soit $\tilde{Z}_{1}, \ldots, \tilde{Z}_{n}$ des v.a. i.i.d. de loi $N(0,1)$. Posons $Y_{i}=n^{-1 / 2} \tilde{Y}_{i}$ et $Z_{i}=n^{-1 / 2} \tilde{Z}_{n}$. Il suffit de montrer que

$$
\left|E\left(g\left(Y_{1}+\cdots+Y_{n}\right)\right)-E\left(g\left(Z_{1}+\cdots+Z_{n}\right)\right)\right| \leq \frac{C}{3 !} \frac{E\left(\left|\tilde{Y}_{1}\right|^{3}\right)+E\left(\left|\tilde{Z}_{1}\right|^{3}\right)}{\sqrt{n}},
$$

où $C=\sup _{x \in \mathbb{R}}\left|g^{(3)}(x)\right|$. Posons

$$
\begin{aligned}
U_{i} & =Y_{1}+\cdots+Y_{i-1}+Y_{i}+Z_{i+1}+\cdots+Z_{n}, \\
V_{i} & =Y_{1}+\cdots+Y_{i-1}+Z_{i+1}+\cdots+Z_{n}
\end{aligned}
$$

(noter l'absence du terme $Y_{i}$ dans la définition de $V_{i}$ ), de sorte que

$$
U_{i}=V_{i}+Y_{i}, \quad U_{i-1}=V_{i}+Z_{i}
$$

et la différence des espérances dans le membre de gauche de (7) est égale à

$$
\begin{aligned}
E\left(g\left(U_{n}\right)\right)-E\left(g\left(U_{0}\right)\right) & =\sum_{i=1}^{n}\left[E\left(g\left(U_{i}\right)\right)-E\left(g\left(U_{i-1}\right)\right)\right] \\
& =\sum_{i=1}^{n}\left[E\left(g\left(V_{i}+Y_{i}\right)\right)-E\left(g\left(V_{i}+Z_{i}\right)\right)\right] .
\end{aligned}
$$

D'après le lemme 2, la valeur absolue de cette expression est majorée par

$$
\sum_{i=1}^{n} \frac{C}{3 !}\left(E\left(\left|Y_{i}\right|^{3}\right)+E\left(\left|Z_{i}\right|^{3}\right)\right)=n \frac{C}{3 !} n^{-3 / 2}\left(E\left(\left|\tilde{Y}_{1}\right|^{3}\right)+E\left(\left|\tilde{Z}_{1}\right|^{3}\right)\right),
$$

ce qui montre (7) et achève la démonstration du lemme 3 .

La troisième étape est de montrer que la conclusion du lemme 3 implique la convergence des fonctions de répartitions.

Proposition 4. Soit $Z$ une v.a. continue et $\left(Z_{n}, n \in \mathbb{N}\right)$ une suite de v.a. On suppose que pour toute fonction $g \in C_{\mathrm{b}}^{3}(\mathbb{R}), \lim _{n \rightarrow \infty} E\left(g\left(Z_{n}\right)\right)=E(g(Z))$. Alors pour tout $x \in \mathbb{R}$, $\lim _{n \rightarrow \infty} F_{Z_{n}}(x)=F_{Z}(x)$.

Démonstration. Soit $x \in \mathbb{R}$ et $k \in \mathbb{N}^{*}$ fixés. Construisons (cf. fig. 1) deux fonctions $\tilde{g}_{k}$ et $g_{k}$ dans $C_{\mathrm{b}}^{3}(\mathbb{R})$ telles que pour tout $z \in \mathbb{R}$,

$$
1_{]-\infty, x-\frac{1}{k}\right]}(z) \leq \tilde{g}_{k}(z) \leq 1_{]-\infty, x]}(z) \leq g_{k}(z) \leq 1_{]-\infty, x+\frac{1}{k}\right]}(z) .
$$

Observons que pour tout $n \in \mathbb{N}$,

$$
F_{Z_{n}}(x)=P\left\{Z_{n} \leq x\right\}=E\left(1_{-\infty, x]}\left(Z_{n}\right)\right) \leq E\left(g_{k}\left(Z_{n}\right)\right),
$$

et donc, d'après l'hypothèse,

$$
\limsup _{n \rightarrow \infty} F_{Z_{n}}(x) \leq \lim _{n \rightarrow \infty} E\left(g_{k}\left(Z_{n}\right)\right)=E\left(g_{k}(Z)\right) \leq E\left(1_{\left.-\infty, x+\frac{1}{k}\right]}(Z)\right)=F_{Z}\left(x+\frac{1}{k}\right) .
$$




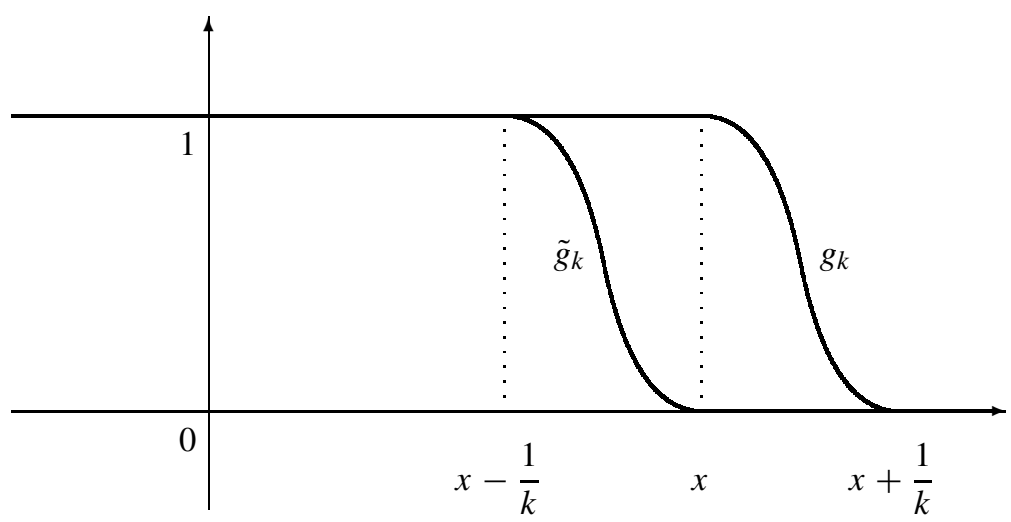

Fig. 1 Esquisse des fonctions $\tilde{g}_{k}$ et $g_{k}$.

De la même manière,

$$
F_{Z_{n}}(x)=E\left(1_{]-\infty, x]}\left(Z_{n}\right)\right) \geq E\left(\tilde{g}_{k}\left(Z_{n}\right)\right),
$$

et donc

$$
\begin{aligned}
\liminf _{n \rightarrow \infty} F_{Z_{n}}(x) & \geq \lim _{n \rightarrow \infty} E\left(\tilde{g}_{k}\left(Z_{n}\right)\right) \\
& =E\left(\tilde{g}_{k}(Z)\right) \geq E\left(1_{]-\infty, x-\frac{1}{k}\right]}(Z)\right)=F_{Z}\left(x-\frac{1}{k}\right) .
\end{aligned}
$$

Nous déduisons de (10) et (11) que pour tout $k \in \mathbb{N}^{*}$,

$$
F_{Z}\left(x-\frac{1}{k}\right) \leq \liminf _{n \rightarrow \infty} F_{Z_{n}}(x) \leq \limsup _{n \rightarrow \infty} F_{Z_{n}}(x) \leq F_{Z}\left(x+\frac{1}{k}\right) .
$$

En faisant tendre $k$ vers l'infini, nous concluons, puisque $F_{Z}$ est continue, que

$$
\liminf _{n \rightarrow \infty} F_{Z_{n}}(x) \text { et } \quad \limsup _{n \rightarrow \infty} F_{Z_{n}}(x)
$$

sont égales et que $\lim _{n \rightarrow \infty} F_{Z_{n}}(x)=F_{Z}(x)$. La proposition 4 est démontrée.

Le travail principal est maintenant fait. Il ne reste plus qu'à tirer partie des trois étapes précédentes.

Démonstration du théorème 1 sous l'hypothèse A. Posons $\tilde{Y}_{n}=\frac{X_{n}-\mu}{\sigma}$. Alors

$$
\frac{S_{n}-n \mu}{\sigma \sqrt{n}}=\frac{\tilde{Y}_{1}+\cdots+\tilde{Y}_{n}}{\sqrt{n}}
$$

et vu l'hypothèse $\mathrm{A},\left(\tilde{Y}_{n}, n \in \mathbb{N}\right)$ vérifie les hypothèses du lemme 3. Soit $Z$ une v.a. $N(0,1)$. D'après la conclusion du lemme $3, Z_{n}=n^{-1 / 2}\left(\tilde{Y}_{1}+\cdots+\tilde{Y}_{n}\right)$ et $Z$ vérifient les hypothèses de la proposition 4 , donc aussi sa conclusion. Le théorème 1 est démontré. 
Remarque 5. La démonstration du lemme 3 est prise dans [12, Teorema 6.2], où M. SanzSolé fait cependant l'hypothèse que les v.a. $\tilde{Y}_{n}$ sont bornées. La proposition 4 est standard [5, Theorem (2.2)].

\section{Estimation de la vitesse de convergence}

Dans cette section, nous présentons un raffinement du théorème 1 qui donne une estimation de la vitesse de convergence de la probabilité dans le membre de gauche de (1) vers le membre de droite.

Soit $g_{0}: \mathbb{R} \rightarrow \mathbb{R}$ une fonction décroissante telle que, pour tout $z \in \mathbb{R}, 1_{]-\infty, 0]}(z) \leq$ $g_{0}(z) \leq 1_{]-\infty, 1]}(z)$ et posons $C^{(3)}=\sup _{z \in \mathbb{R}}\left|g_{0}^{(3)}(z)\right|$. Soit $\Phi(\cdot)$ la fonction de répartition d'une v.a. $Z$ de loi $N(0,1)$.

Proposition 6. Avec les hypothèses et notations du théorème 1 et sous l'hypothèse A,

$$
\left|P\left\{\frac{S_{n}-n \mu}{\sigma \sqrt{n}} \leq x\right\}-\Phi(x)\right| \leq n^{-1 / 8}\left[\frac{C^{(3)}}{3 !}\left(E\left(\frac{\left|X_{1}\right|^{3}}{\sigma^{3}}\right)+E\left(|Z|^{3}\right)\right)+\frac{1}{\sqrt{2 \pi}}\right] .
$$

Démonstration. Pour $k \in \mathbb{N}^{*}$ et $z \in \mathbb{R}$, posons $g_{k, x}(z)=g_{0}(k(z-x))$ et $\tilde{g}_{k, x}(z)=$ $g_{0}\left(k\left(z-x+\frac{1}{k}\right)\right)$. Observons que (9) est satisfaite si $\tilde{g}_{k}$ et $g_{k}$ sont respectivement remplacés par $\tilde{g}_{k, x}$ et $g_{k, x}$. De plus

$$
\sup _{z \in \mathbb{R}}\left|g_{k, x}^{(3)}(z)\right| \leq C^{(3)} k^{3}, \quad \sup _{z \in \mathbb{R}}\left|\tilde{g}_{k, x}^{(3)}(z)\right| \leq C^{(3)} k^{3}
$$

et

$$
\begin{aligned}
& 0 \leq g_{k, x}(z)-1_{]-\infty, x]}(z) \leq 1_{] x, x+\frac{1}{k}\right]}(z), \\
& -1_{] x-\frac{1}{k}, x\right]}(z) \leq \tilde{g}_{k, x}(z)-1_{]-\infty, x]}(z) \leq 0 .
\end{aligned}
$$

Soit $U_{n}=(\sigma \sqrt{n})^{-1}\left(S_{n}-n \mu\right)$. D'après (9),

$$
F_{U_{n}}(x)-\Phi(x) \leq E\left(g_{k, x}\left(U_{n}\right)\right)-E\left(g_{k, x}(Z)\right)+E\left(g_{k, x}(Z)-1_{]-\infty, x]}(Z)\right) .
$$

D’après (7), la différence des deux premiers termes du membre de droite est majorée par

$$
\frac{C^{(3)} k^{3}}{3 !} n^{-1 / 2}\left(E\left(\frac{\left|X_{1}\right|^{3}}{\sigma^{3}}\right)+E\left(|Z|^{3}\right)\right)
$$

et, d'après (13), le dernier terme est majoré par

$$
P\left\{x<Z \leq x+\frac{1}{k}\right\}=\frac{1}{\sqrt{2 \pi}} \int_{x}^{x+\frac{1}{k}} e^{-u^{2} / 2} d u \leq \frac{1}{k \sqrt{2 \pi}} .
$$

En posant $k=n^{1 / 8}$ (qui est le meilleur choix), on obtient (12) sans les valeurs absolues. D'après (9) à nouveau,

$$
F_{U_{n}}(x)-\Phi(x) \geq E\left(\tilde{g}_{k, x}\left(U_{n}\right)\right)-E\left(\tilde{g}_{k, x}(Z)\right)+E\left(\tilde{g}_{k, x}(Z)-1_{]-\infty, x]}(Z)\right) .
$$


En procédant exactement comme ci-dessus, on obtient que

$$
F_{U_{n}}(x)-\Phi(x) \geq-\frac{C^{(3)} k^{3}}{3 !} n^{-1 / 2}\left(E\left(\frac{\left|X_{1}\right|^{3}}{\sigma^{3}}\right)+E\left(|Z|^{3}\right)\right)-\frac{1}{k \sqrt{2 \pi}},
$$

ce qui achève la démonstration.

Remarque 7. Ce résultat se trouve déjà dans Lindeberg [9]. La borne en $n^{-1 / 8}$ n'est pas optimale, le meilleur résultat étant celui de Berry et Esseen en $n^{-1 / 2}$ [5, Sec. 2.4].

\section{Démonstration du théorème 1 sans l'hypothèse $\mathrm{A}$}

Dans cette section, nous présentons une variante de la démonstration exposée dans la Section 2 qui permet de s'affranchir de l'hypothèse A. Il s'agit principalement de mieux gérer le reste qui provient de la formule de Taylor.

Lemme 8. Soit $g \in C_{\mathrm{b}}^{3}(\mathbb{R}), V, Y$ et $Z$ trois v.a. indépendantes telles que $E(Y)=$ $E(Z), E\left(Y^{2}\right)=E\left(Z^{2}\right)$ et $E\left(|Z|^{3}\right)<+\infty$. Posons $C^{(2)}=\sup _{x \in \mathbb{R}}\left|g^{\prime \prime}(x)\right|$ et $C^{(3)}=$ $\sup _{x \in \mathbb{R}}\left|g^{(3)}(x)\right|$. Alors pour tout $\varepsilon \in[0, \infty]$,

$$
\begin{aligned}
|E(g(V+Y))-E(g(V+Z))| \leq & \frac{C^{(3)}}{2} E\left(|Y|^{3} 1_{\{|Y|<\varepsilon\}}\right) \\
& +C^{(2)} E\left(|Y|^{2} 1_{\{|Y| \geq \varepsilon\}}\right)+\frac{C^{(3)}}{2} E\left(|Z|^{3}\right) .
\end{aligned}
$$

Démonstration. D'après la formule de Taylor d'ordre 1,

$$
\begin{aligned}
g(x+h) & =g(x)+g^{\prime}(x) h+\int_{x}^{x+h}(x+h-t) g^{\prime \prime}(t) d t \\
& =g(x)+g^{\prime}(x) h+\frac{1}{2} g^{\prime \prime}(x) h^{2}+\tilde{R}_{1}(x, h),
\end{aligned}
$$

où

$$
\tilde{R}_{1}(x, h)=\int_{x}^{x+h}(x+h-t)\left(g^{\prime \prime}(t)-g^{\prime \prime}(x)\right) d t .
$$

En particulier,

$$
\left|\tilde{R}_{1}(x, h)\right| \leq \frac{|h|^{2}}{2} \sup _{x \leq t \leq x+h}\left|g^{\prime \prime}(t)-g^{\prime \prime}(x)\right| \leq \min \left(\frac{|h|^{3}}{2} C^{(3)}, h^{2} C^{(2)}\right) .
$$

On reprend la démonstration du lemme 2 jusqu'à (5) et on obtient (à la place de (6))

$$
|E(g(V+Y))-E(g(V+Z))| \leq E\left(\left|\tilde{R}_{1}(V, Y)\right|\right)+E\left(\left|\tilde{R}_{1}(V, Z)\right|\right) .
$$

Or, le deuxième terme du membre de droite est majoré par $\frac{C^{(3)}}{2} E\left(|Z|^{3}\right)$, alors que le premier est majoré par

$$
\frac{C^{(3)}}{2} E\left(|Y|^{3} 1_{\{|Y|<\varepsilon\}}\right)+C^{(2)} E\left(Y^{2} 1_{\{|Y| \geq \varepsilon\}}\right) .
$$

Le lemme 8 est démontré. 
Lemme 9. Soit $\left(\tilde{Y}_{n}, n \in \mathbb{N}\right)$ une suite de v.a. i.i.d. telles que $E\left(\tilde{Y}_{1}^{2}\right)=1$ et $E\left(\tilde{Y}_{1}\right)=0$. Soit $\tilde{Z}$ une v.a. $N(0,1)$. Alors pour toute fonction $g \in C_{\mathrm{b}}^{3}(\mathbb{R})$,

$$
\lim _{n \rightarrow \infty} E\left(g\left(\frac{\tilde{Y}_{1}+\cdots+\tilde{Y}_{n}}{\sqrt{n}}\right)\right)=E(g(\tilde{Z})) .
$$

Démonstration. Soit $\varepsilon>0$. On reprend les notations de la démonstration du lemme 3 . La majoration (8) de $E\left(g\left(U_{n}\right)\right)-E\left(g\left(U_{0}\right)\right)$ utilise maintenant le lemme 8 et devient

$$
\begin{array}{r}
\sum_{i=1}^{n}\left[\frac{C^{(3)}}{2} E\left(\left|Y_{i}\right|^{3} 1_{\left\{\left|Y_{i}\right|<\varepsilon\right\}}\right)+C^{(2)} E\left(Y_{i}^{2} 1_{\left\{\left|Y_{i}\right| \geq \varepsilon\right\}}\right)+\frac{C^{(3)}}{2} E\left(\left|Z_{i}\right|^{3}\right)\right] \\
=\sum_{i=1}^{n}\left[\frac{C^{(3)}}{2} n^{-3 / 2} E\left(\left|\tilde{Y}_{i}\right|^{3} 1_{\left\{\left|\tilde{Y}_{i}\right|<n^{1 / 2} \varepsilon\right\}}\right)+C^{(2)} n^{-1} E\left(\tilde{Y}_{i}^{2} 1_{\left\{\left|\tilde{Y}_{i}\right| \geq n^{1 / 2} \varepsilon\right\}}\right)\right. \\
\left.+\frac{C^{(3)}}{2} n^{-3 / 2} E\left(\left|\tilde{Z}_{i}\right|^{3}\right)\right] .
\end{array}
$$

Puisque $\left|\tilde{Y}_{i}\right|^{3} 1_{\left\{\left|\tilde{Y}_{i}\right|<n^{1 / 2} \varepsilon\right\}} \leq n^{1 / 2} \varepsilon \tilde{Y}_{i}^{2}$ et $E\left(\tilde{Y}_{i}^{2}\right)=1$, cette expression est majorée par

$$
\varepsilon \frac{C^{(3)}}{2}+C^{(2)} E\left(\tilde{Y}_{1}^{2} 1_{\left\{\left|\tilde{Y}_{1}\right| \geq n^{1 / 2} \varepsilon\right\}}\right)+\frac{C^{(3)}}{2} n^{-1 / 2} E\left(\left|\tilde{Z}_{1}\right|^{3}\right) .
$$

Lorsque $n \rightarrow \infty$, le deuxième terme tend vers 0 d'après le théorème de la convergence monotone et le troisième terme tend aussi vers 0 . Puisque $\varepsilon>0$ est arbitraire, le lemme 9 est démontré.

Démonstration du théorème 1 . Posons $\tilde{Y}_{n}=\frac{X_{n}-\mu}{\sigma}$. Alors

$$
\frac{S_{n}-n \mu}{\sigma \sqrt{n}}=\frac{\tilde{Y}_{1}+\cdots+\tilde{Y}_{n}}{\sqrt{n}}
$$

et $\left(\tilde{Y}_{n}, n \in \mathbb{N}\right)$ vérifie les hypothèses du lemme 9 . Soit $Z$ une v.a. $N(0,1)$. D'après la conclusion du lemme $9, Z_{n}=n^{-1 / 2}\left(\tilde{Y}_{1}+\cdots+\tilde{Y}_{n}\right)$ et $Z$ vérifient les hypothèses de la proposition 4 , donc aussi sa conclusion. Le théorème 1 est démontré.

\section{Remarque 10.}

(a) Cette démonstration est aussi due à Lindeberg [9]. Elle m'a été communiquée par Ch.-E. Pfister. Elle se trouve par exemple dans [2, Chap. 1.7] et [6, p. 262-263], mais dans ces références, elle est précédée par de nombreuses considérations qui font appel à la théorie de la mesure (à noter que Feller attribue incorrectement certaines idées de la preuve à H.F. Trotter).

(b) Remarquons que la convergence ponctuelle de fonctions de répartition, qui sont monotones et uniformément bornées, implique la convergence uniforme de ces fonctions. 
(c) Sans l'hypothèse A, on ne peut estimer la vitesse de convergence: la méthode de la proposition 6 donne cependant l'estimation suivante:

$$
\begin{aligned}
\left|F_{U_{n}}(x)-\Phi(x)\right| \leq & \varepsilon \frac{C^{(3)} k^{3}}{2}+C^{(2)} k^{2} E\left(\frac{X_{1}^{2}}{\sigma^{2}} 1_{\left\{\left|X_{1}\right|>\sigma n^{1 / 2} \varepsilon\right\}}\right) \\
& +\frac{C^{(3)} k^{3}}{2} n^{-1 / 2} E\left(|Z|^{3}\right)+\frac{1}{k \sqrt{2 \pi}} .
\end{aligned}
$$

En faisant d'abord $n \rightarrow \infty$, puis $\varepsilon \downarrow 0$, puis $k \rightarrow \infty$, on déduit bien le théorème 1 .

Addendum. D. Khoshnevisan m'a signalé que Lindeberg [9] attribue l'idée de la preuve du lemme 3 à A. Liapounoff, cf. "Sur une proposition de la théorie des probabilités", Bull. Acad. Sci. St. Petersbourg 13 (1900), 359-386, et "Nouvelle forme du théorème sur la limite de probabilité", Mém. Acad. Sci. St. Petersbourg 12 (1901), 1-24. Cependant, Lindeberg [9] exploite cette idée en particulier pour démontrer le théorème 1 sans autre hypothèse que l'existence du deuxième moment.

\section{Références}

[1] Beintkus, V.; Götze, F.; Paulauskas, V.; Rackauskas, A.: The accuracy of Gaussian approximation in Banach spaces. In: Prokhorov, Yu.V.; Statulevicius, V. (eds.): Limit theorems of probability theory. Springer, 2000, 25-111

[2] Billingsley, P.: Convergence of Probability Measures. Wiley, 1968.

[3] Chatterji, S.D.: Comments on Hausdorff's work in probability theory. In: Gesammelte Werke von F. Hausdorff, Vol. 5. Springer, 2005, to appear.

[4] Durrett, R.: The Essentials of Probability. Duxbury Press, 1994.

[5] Durrett, R.: Probability: Theory and Examples. (2nd ed.). Duxbury Press, 1996.

[6] Feller, W.: An Introduction to Probability Theory and its Applications. Vol. 2 (2nd ed.). Wiley, 1971.

[7] Krengel, U.: Einführung in die Wahrscheinlichkeitstheorie und Statistik. Vieweg, 2000.

[8] Larsen. R.; Marx, M.: Introduction to Mathematical Statistics and its Applications. Prentice Hall, 1986.

[9] Lindeberg, J.: Eine neue Herleitung des Exponentialgesetzes in der Wahrscheinlichkeitsrechnung. Math. Z. 15 (1922), 211-225.

[10] Petrov, V.V.: Classical-type limit theorems for sums of independent random variables. In: Prokhorov, Yu.V.; Statulevicius, V. (eds.): Limit theorems of probability theory. Springer, 2000, 1-24.

[11] Ross, S.M.: A First Course in Probability. MacMillan, 1994.

[12] Sanz i Solé, M.: Probabilitats. Edicions de la Universitat de Barcelona, 1999.

[13] Stirzaker, D.: Elementary Probability. (2nd ed.). Cambridge University Press, 2003.

\section{Robert C. Dalang}

Institut de Mathématiques

Ecole Polytechnique Fédérale

Station 8

CH-1015 Lausanne, Switzerland

e-mail: robert.dalang@epfl.ch 